\title{
IN-PLANE MEMS-BASED FABRY-PEROT FILTER FOR HIGH-SPEED WAVELENGTH-SWEPT SEMICONDUCTOR LASER

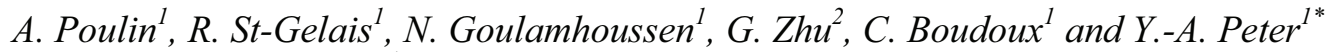 \\ ${ }^{1}$ Department of Engineering Physics \\ ${ }^{2}$ Department of Electrical Engineering \\ Ecole Polytechnique de Montréal, Montréal (Québec) H3C 3A7 CANADA
}

\begin{abstract}
This paper reports the development of a MEMS-based wavelength-swept semiconductor laser for biomedical imaging. MEMS-based sources present numerous advantages over the ones based on polygon-scanners. Their non-linearity is however an important drawback for the development of medical applications such as optical coherence tomography where video rate imaging is of great importance. The device we propose overcomes this problem. Its in-plane design enables the push-pull configuration resulting in a linear behavior. Experimental results demonstrate the linearity of the spectral sweep for frequencies up to $30 \mathrm{kHz}$. The laser, centered at $1310 \mathrm{~nm}$, was tuned over more than $20 \mathrm{~nm}$.
\end{abstract}

\section{INTRODUCTION}

Rapidly tunable wavelength-swept sources are of particular interest for optical coherence tomography (OCT). OCT is a technique which consists in acquiring by optical interrogation a series of parallel dept-scans within a semi-transparent medium. The scans are then synthesized in a three dimensional model from which cross sectional views can be extracted. OCT is mainly used as a medical imaging and diagnosis tool [1]. The main advantages over x-rays and ultrasound imagery are its resolution, speed, size and cost. OCT provides information on tissue architecture, but its resolution does not typically allow visualization of subcellular structures. This information is however precious for medical diagnosis of cancers and as a real-time feedback for surgical oncologist [2].

The development of new applications in oncology, and in other fields such as cardiology and gastroenterology, requires OCT systems with real-time imaging capability. The first OCT techniques, based on time-domain low coherence interferometry, required mechanical scanning of a reference arm which limited the acquisition speed. With the development of Fourier-domain OCT techniques, the reference arm remains fixed. Interferometric detection is either performed through a spectrometer or by rapidly tuning the wavelength of the source - a technique called sweptsource (SS) OCT. The reflectivity profile as a function of depth is then recovered by Fourier transforming the detected signal. Spectral scan or spectrometric measurement can be achieved much faster than the equivalent mechanical scan. SS-OCT therefore enables video rate imaging and paves the way for an array of new medical applications. The acquisition rate of OCT images is limited by the sweeping speed of the source and the data treatment needed for image reconstruction. Consequently, the post acquisition treatment must be straightforward and avoid costly steps such as interpolation for relinearization of the spectrum sweep. For these reasons, a laser exhibiting a truly linear sweep over the spectrum at high sweeping rate is of great interest for OCT medical applications.

Various swept-sources have been developed for this specific field [3]-[6]. One of the most challenging aspects has been to reach tuning speeds of tens of $\mathrm{kHz}$. It has first been achieved with sources using a polygon-scanner based filter [3]. This filter exhibits a highly linear response and can achieve tuning speeds faster than $100 \mathrm{kHz}$ [7]. However, the polygon scanner is bulky, expensive, complex to fabricate and involves a free space component prone to misalignments. These drawbacks limit the accessibility of the technology and consequently slow down its use in the development of new medical applications. To address this issue, sources based on micro-electromechanical systems (MEMS) have been developed [4]-[6]. MEMS filters or reflectors are well suited for external-cavity tunable lasers and provide many benefits [8]. They exhibit small dimensions, low fabrication cost and simple designs. They minimize the required optical alignments and the cavity length. In most cases, devices are based on out-ofplane designs [4], [5]. In comparison with in-plane designs, this configuration makes the optical alignment more complex and less robust. In addition, it often involves parallel plate actuators that are intrinsically non-linear and present risks of pull-in. A first inplane MEMS-based swept source was recently demonstrated [6]. The authors approach, based on the Vernier effect, could allow a linear spectral scan at high frequency. However, it involves multiple optical components and requires a complex electronic control. Moreover, the Vernier effect can allow stimulated emission at unwanted resonance orders and therefore generate important instabilities in the laser.

The swept-source design we propose combines the advantages of both polygon and MEMS filters. A tunable FabryPerot (FP) is used in the laser cavity as a wavelength selector. This MEMS filter, unlike the ones used in [4] and [5], is based on an inplane design that provides many benefits. This original design allows the use of comb-drive actuators that do not exhibit pull-in voltage. Moreover, it allows including a third electrode to control the actuator in push-pull configuration [9]. This configuration is used to produce a linear behavior of the comb-drive actuators and overcome the MEMS non-linearity issue. The laser presented in [6] is also based on an in-plane design. The wavelength selection is achieved with a combination of integrated filters and phase modulators. The fabrication process is however complex. It involves multiple materials, deposition and etching steps. The technique we propose is based on a single free-space tunable filter. It requires only one photolithography step and materials are limited to a Silicon On Insulator (SOI) substrate. The in-plane approach we propose was inspired by our previous work on tunable fiber laser for optical communication systems [10]. The filter and the laser designs are however significantly different.

\section{FILTER DESIGN}

The fabricated filter is shown in Fig.1. It is composed of two main components, which are the Fabry-Perot cavity and the actuator. The inset of Fig.1 corresponds to a close-up view of the FP cavity. Two Bragg mirrors are formed by a series of alternating silicon and air layers. Combined together, coplanar and separated by an air gap, they form the FP resonator. This type of cavity, inside the reflection bandwidth of the Bragg mirrors, reflects light except at its resonance frequencies where the transmission is maximal. It can therefore be used as a wavelength selection filter. Various parameters influence the optical response and the performances of such filters. Our design was optimized using a model recently published, taking into account not only the 


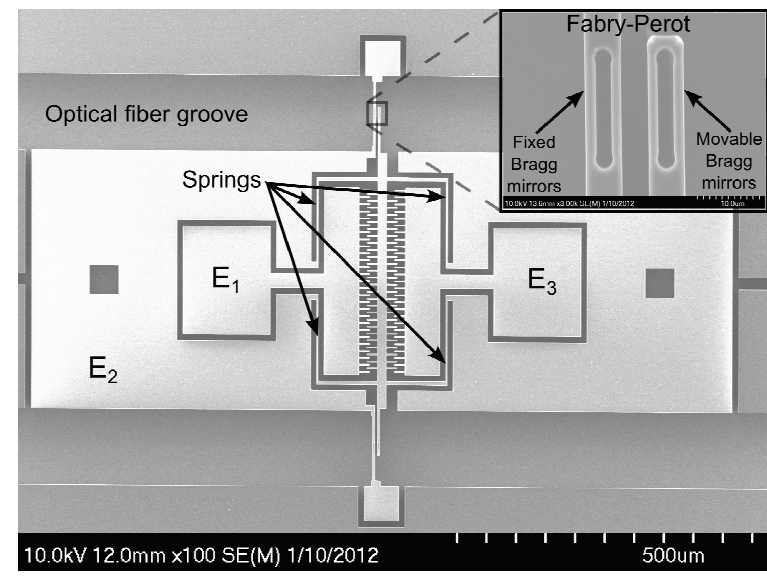

Figure 1: Scanning Electron Microscopy image of the fabricated device. The substrate is a Silicon on Insulator wafer with a $70 \mu \mathrm{m}$ thick device layer.

dimensions and refractive indices of the layers but also the limitations due to Gaussian beam divergence and surface roughness [11].

The second element of importance, the actuator, is composed of two interdigited combs and three electrodes referred as $\mathrm{E}_{1}, \mathrm{E}_{2}$ and $\mathrm{E}_{3}$. The electrode $\mathrm{E}_{2}$, connected to the central suspended section, is grounded. The suspended section is attached to one of the FP Bragg mirrors, which displacement allows spectral tuning of the filter. The use of comb-drive actuators was enabled by the in-plane design. Unlike parallel plate actuators, whose plates collapse for displacements larger than one third of the initial gap, these later allow large displacements and do not exhibit pull-in voltage.

Typical actuators are composed of two electrodes. An alternative voltage $\mathrm{V}_{\mathrm{a}}$ is applied on the fixed electrode while the movable one is grounded. For example, referring at the Fig.1, the electrodes $E_{2}$ would be grounded while the electrode $E_{1}$ would be set at $\mathrm{V}_{\mathrm{a}}$. This type of actuation leads to a quadratic relation between the displacement and the applied voltage. In order to maximize the performance for OCT applications, we look for a device providing a linear scan over the covered spectrum. A linear behavior can be obtained from comb-drive actuators by working in push-pull configuration and by the mean of an additional electrode. It is interesting to note that this third electrode could not be implemented in the design of out-of-plane filters. In the push-pull configuration the movable electrode is surrounded by two electrodes. A bias source $V_{b}$ and an alternative source $V_{a}$ are used to drive the combs. The applied voltage on $\mathrm{E}_{1}$ and $\mathrm{E}_{3}$ are respectively $\mathrm{V}_{\mathrm{b}}+\mathrm{V}_{\mathrm{a}}$ and $\mathrm{V}_{\mathrm{b}}-\mathrm{V}_{\mathrm{a}}$ while $\mathrm{E}_{2}$ is grounded. The value of the bias source determines the slope of the linear relation observed between the displacement and the voltage.

The optimization of the design was not limited to the optical performances of the resonator. The electromechanical response of the device was simulated and optimized using the finite element method (FEM). One of the aspects studied was the frequency and shape of the first resonance mode. We wanted the natural resonance mode to be an in-plane oscillation, parallel to the optical axis of the cavity. We designed the springs in order to get the natural frequency at $40 \mathrm{kHz}$. Figure 2 presents the natural resonance mode of the device obtained through FEM simulation. We can see that its shape corresponds to the one desired. The shape of the first mode was mainly determined by the symmetry of the device with respect to the $\mathrm{x}$ axis and the ratio between its

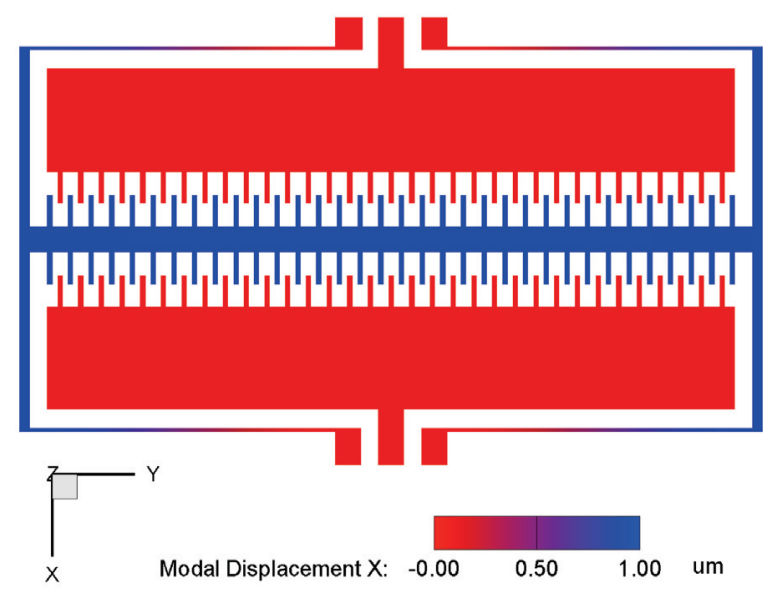

Figure 2: Natural vibration mode of the actuator obtained by finite element simulation. The movement is strictly limited to the $x$ axis.

spring constant along the $\mathrm{z}$ and the $\mathrm{x}$ axis. The stiffness of the central beam has to be high enough in order to avoid its deformation when actuated at high frequencies.

Details of the fabrication process will not be presented in this work. Further information can be found in [10]. It is however important to mention that the device is structured by bulk micromachining of a silicon on insulator substrate and only one photolithography step is required during the process. The original in-plane design enables this simple fabrication process. In addition, it allows the integration of optical fiber grooves which greatly simplify the optical alignment.

\section{LASER DESIGN}

A schematic of the laser design is shown in Fig.3. The gain medium is a semiconductor optical amplifier (SOA) from Thorlabs (BOA1017S, Newton, NJ) centered at $1310 \mathrm{~nm}$ with a spontaneous emission $3 \mathrm{~dB}$ bandwidth of $70 \mathrm{~nm}$. The performance of the SOA was found to be highly influenced by polarization. This component is therefore surrounded by two polarization controllers (PC) which were used to optimize the laser output power. Two circulators are used as isolators in order to avoid back reflections from the different interfaces to propagate in the cavity to the gain medium. These retroreflections could stimulate emission at unwanted frequencies and alter the laser emission spectrum.

The laser cavity is composed of a SOA, two polarization

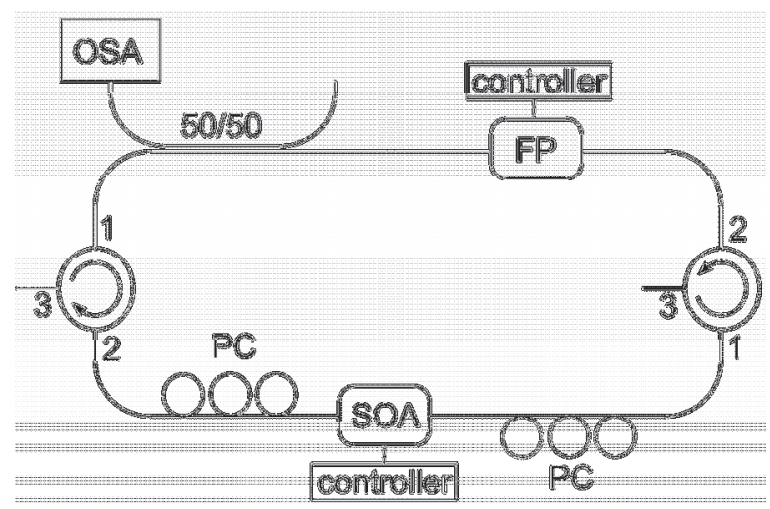

Figure 3: Scheme of the laser setup, connected to an optical spectrum analyzer for characterization. 
controllers, two isolators and the MEMS-based Fabry-Perot filter. All these components are optically connected using optical fiber (Corning SMF-28) in a ring configuration cavity. The gain medium emits a spontaneous emission spectrum which propagates through the cavity in the direction determined by the two isolators. The tunable FP is used to filter this spontaneous emission. It only allows wavelengths near its resonance to complete multiple roundtrips in the cavity, stimulate emission in the gain medium and consequently produce a laser emission. A 50/50 coupler was inserted in the ring cavity to create the laser output. Finally, an optical spectrum analyzer (OSA) was used at the laser output for characterization purposes.

\section{RESULTS}

Using the laser design shown in Fig.3, the fabricated device was used as an intra-cavity filter to form a wavelength sweptsource. The laser, centered at $1310 \mathrm{~nm}$, was tuned over more than $20 \mathrm{~nm}$ as shown in Fig.4. The full-width at half-maximum is lower than $0.06 \mathrm{~nm}$. Its measurement was limited by the resolution bandwidth of the OSA. At this stage, the main limitation of the laser for OCT application is its small tuning range. The tuning range could be increased by a factor of three with the optimization of the fabrication process. Previous work reported in [10] demonstrates that such performances can indeed be reached. Another approach would be to use multiple filters in parallel, covering specific and complementary spectral region. We could therefore expect to cover the whole spectrum of the SOA. The low cost, small size and easy integration of the filter enable this approach.

A white light interferometer (Fogale Nanotech Photomap 3D) was used to characterize the actuator. Its in-plane displacement was monitor for different driving voltage amplitudes and frequencies. First, we applied an alternative voltage between the electrode E1 and E2. The amplitude of the movable Bragg mirror motion was measured in function of the alternative voltage frequency. The results are reported in Fig.5. The natural vibration frequency was observed at $35 \mathrm{kHz}$. It is approximately $13 \%$ lower than the expected value of $40 \mathrm{kHz}$. This shift can be explained by slight variations between the expected and the experimental dimensions of the springs. These variations mainly come from the etching step where we can observe some under-etch and a slight non-verticality of the trenches. We believe these geometrical parameters have a greater influence than the air damping, which have been taken into account during the optimization of the device geometry. It was confirmed by visual inspection that the vibration mode corresponding to this frequency is the one shown in Fig.2. The resonance peak of this vibration mode is narrow and does not affect frequencies lower than $30 \mathrm{kHz}$. The device should consequently be able to follow arbitrary signal waveform with

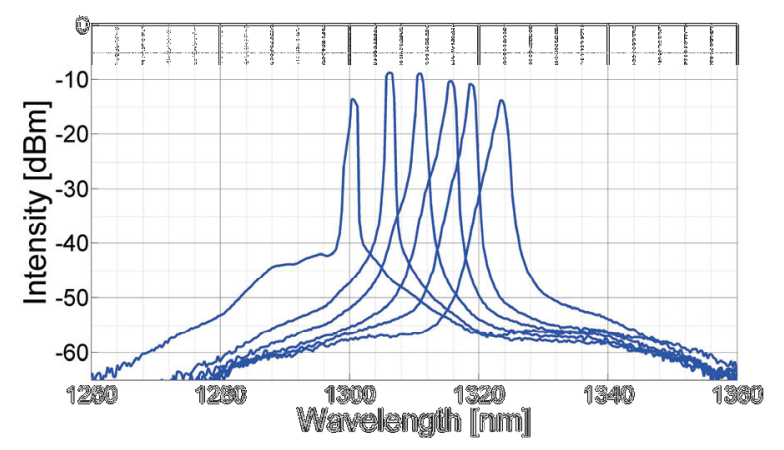

Figure 4: Spectral tuning of the laser over more than $20 \mathrm{~nm}$.

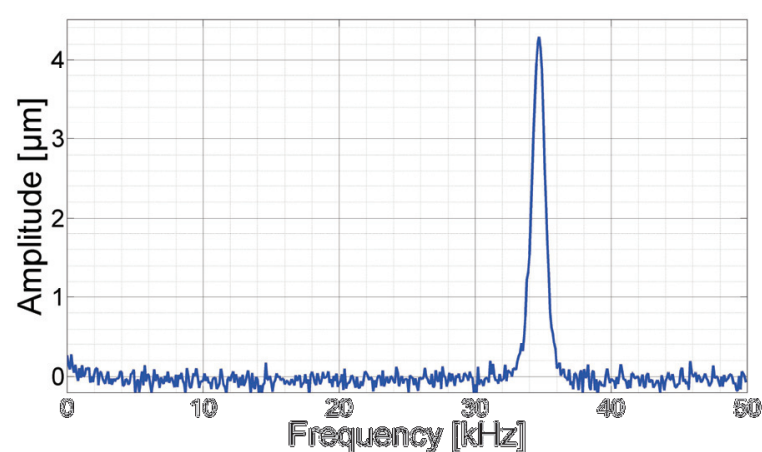

Figure 5: Measured amplitude of the Bragg mirror motion for increasing actuation frequency. The natural vibration mode frequency of the actuator is $35 \mathrm{kHz}$. The actuator must be used offresonance (i.e. below $30 \mathrm{kHz}$ ) to follow non-sinusoidal signals.

frequency component lower than $30 \mathrm{kHz}$. This limit on the actuation frequency could easily be overcome by stiffening the springs supporting the movable combs.

The second step in the characterization of the actuator was to study its in-plane motion in function of the applied voltage. Figure 6 reports the experimental results obtained for both the conventional $\left(\mathrm{E}_{1}=\mathrm{V}, \quad \mathrm{E}_{2}=\mathrm{E}_{3}=0, \quad \mathrm{~V}=2 \mathrm{~V}_{\mathrm{a}}\right)$ and the push-pull $\left(\mathrm{E}_{1}=\mathrm{V}_{\mathrm{b}}+\mathrm{V}_{\mathrm{a}}, \mathrm{E}_{2}=0, \mathrm{E}_{3}=\mathrm{V}_{\mathrm{b}}-\mathrm{V}_{\mathrm{a}}\right)$ configurations. In the push-pull mode $\mathrm{V}_{\mathrm{b}}$ was equal to $200 \mathrm{~V}$. The dots correspond to the experimental results. The lines respectively correspond to a linear and a quadratic fit for the push-pull and the conventional configurations. The results clearly demonstrate that a linear behavior can be obtained from comb-drive actuators by working in push-pull configuration.

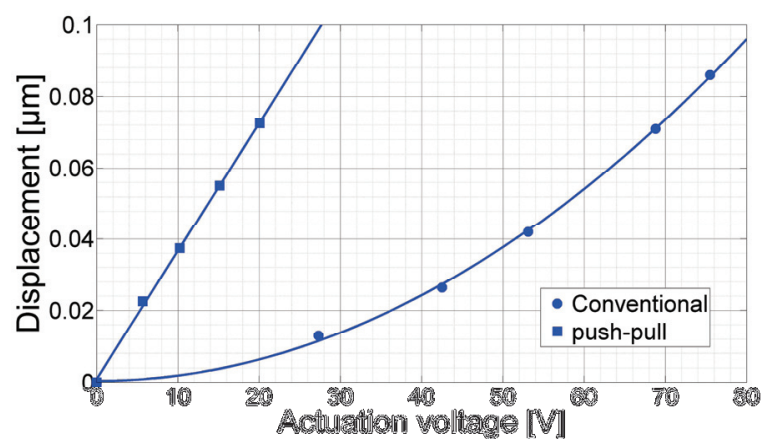

Figure 6: Experimental measurements of the displacement as a function of the applied voltage. Both the push-pull and the conventional actuation techniques were tested.

\section{CONCLUSION}

The device we presented combines the small size, low cost, design simplicity and robustness of MEMS-based sources with the linearity of polygon-scanner based sources. We demonstrated the linearity of the spectral sweep and the $35 \mathrm{kHz}$ natural resonance frequency of the actuator. We can expect the swept-source to follow any arbitrary signal waveform with frequency components lower than $30 \mathrm{kHz}$. This frequency could be easily increased by simple modifications of the device geometry. The laser tuning range, limited to $20 \mathrm{~nm}$, could be further improved by working on the fabrication process or using multiple filters in parallel. We believe the development of a source exhibiting high speed, linearity and low cost is a significant step toward broader uses of OCT for clinical applications. 


\section{REFERENCES}

[1] A. F. Fercher, W. Drexler, C. K. Hitzenberger, and T. Lasser, "Optical coherence tomography - principles and applications", Reports on Progress in Physics, 66, (2003).

[2] J. M. Zara, and C. A. Lingley-Papadopoulos, "Endoscopic OCT Approaches Toward Cancer Diagnosis", Journal of Selected Topics in Quantum Electronics, 14, 1, (2008).

[3] S. H. Yun, C. Boudoux, G. J. Tearney, and B. E. Bouma, "High-speed wavelength-swept semiconductor laser with a polygon-scanner-based wavelength filter", Optics Letters, 28, 20, (2003).

[4] M. Kuznetsov, W. Atia, B. Johnson, and D. Flanders, "Compact Ultrafast Reflective Fabry-Perot Tunable Lasers For OCT Imaging Applications", Proceedings of SPIE: Optical Coherence and Coherence Tomography and Coherence Domain Optical Methods in Biomedicine XIV, (2009), p. 75541F.

[5] K. Isamoto, K. Totsuka, T. Sakai, T. Suzuki, A. Morosawa, C. Chong, H. Fujita, and H. Toshiyoshi, "A High Speed MEMS Scanner for 140-kHz SS-OCT", International Conference on Optical MEMS and Nanophotonics, (2011), pp. 73-74.
[6] B. W. Tilma, Y. Jiao, J. Kotani, B. Smalbrugge, H. P. M. M. Ambrosius, P. J. Thijs, X. J. M. Leijtens, R. Nötzel, M. K. Smit, and E. A. J. M. Bente, "Integrated Tunable QuantumDot Laser for Optical Coherence Tomography in the $1.7 \mu \mathrm{m}$ Wavelength Region", IEEE Journal of Quantum Electronics, 48, 2, (2012).

[7] W. Y. Oh, S. H. Yun, G. J. Tearney, and E. Bouma, " $115 \mathrm{kHz}$ tuning repetition rate ultrahigh-speed wavelength-swept semiconductor laser", Optics Letters, 30, 23, (2005).

[8] A. Q. Liu and X. M. Zhang, "A review of MEMS externalcavity tunable lasers", Journal of Micromechanics and Microengineering, 17, (2007).

[9] W. C. Tang, T.-C. H. Nguyen, and R. T. Howe, "Laterally Driven Polysilicon Resonant Microstructures", IEEE Microelectromechanical Systems Workshop Tech. Dig., (1989), pp. 53-59.

[10] J. Masson, R. St-Gelais, A. Poulin, and Y.-A. Peter, Tunable "Fiber Laser Using a MEMS-Based In Plane Fabry-Pérot Filter", IEEE J. Quantum Electron. 49, 9, (2010).

[11] R. St-Gelais, A. Poulin, and Y.-A. Peter, "Advances in Modeling, Design, and Fabrication of Deep-Etched Multilayer Resonators", Journal of Lightwave Technology, doi: 10.1109/JLT.2012.2191136.

\section{CONTACT}

*Y.-A. Peter, tel: +1-514-340-4711; yves-alain.peter@polymtl.ca 